\title{
El deber de consulta pública previa a la expedición de reglamentos en el régimen jurídico colombiano**
}

\section{Public and previous consultation on administrative rulemaking in the Colombian legal system}

SUMARIO

Introducción. 1. La consulta pública de los reglamentos en el derecho comparado. 2. La consulta pública en el marco normativo vigente en Colombia. 3. La consulta pública en la más reciente jurisprudencia del Consejo de Estado. 3.1. Sentencia del 4 de febrero de 2016, proceso con radicado 11001-0324000-2010-00329-00, C.P.: Guillermo Vargas Ayala, Sección Primera del Consejo de Estado. 3.2. Auto del 15 de febrero de 2016, proceso con radicación 11001-03-27-000-2016-00008-00, M. Sustanciador: Hugo Fernando Bastidas Bárcenas, Sección Cuarta del Consejo de Estado. 3.3. Sentencia del 21 de julio de 2016, proceso con radicado 11001-03-28-000-2015-0003000, C.P.: Alberto Yepes Barreiro, Sección Quinta del Consejo de Estado. 4. Propuestas de actuación e interpretación de la consulta pública a partir del marco normativo vigente. Conclusiones. Referencias.

\section{RESUMEN}

El presente artículo aborda la figura de la consulta pública y previa a la expedición de reglamentos en el sistema jurídico colombiano. Para ello recurre al derecho comparado, a una interpretación sistemática del régimen jurídico

\footnotetext{
* Abogado, politólogo y especialista en Derecho Público de la Universidad EAfrT (Medellín, Colombia). Magíster en Derecho Constitucional del CEPC y LL.M. en Estudios Jurídicos Internacionales de American University, Washington D.C. Profesor asistente del Área de Derecho Público e investigador de la Escuela de Derecho, Universidad EAfIT (Medellín, Colombia). Contacto: agomezv1@eafit.edu.co

** Este artículo hace parte de los resultados parciales del proyecto de investigación titulado "La 'colaboración armónica' interorgánica en Colombia como presupuesto para el cumplimiento de los fines del Estado. Construcción de un marco conceptual y aplicación a un estudio de caso", desarrollado por el Grupo Derecho y Poder de la Escuela de Derecho de la Universidad EAFIT de Medellín-Colombia con financiación interna.

Recibido el 26 de septiembre de 2016, aprobado el 1. ${ }^{\circ}$ de marzo de 2017.

Para citar el artículo: GómEz VeLÁsquEz, A. El deber de consulta pública previa a la expedición de reglamentos en el régimen jurídico colombiano. Derecho del Estado . $^{\circ} 38$, Universidad Externado de Colombia, enero-junio de 2017, pp. 97-125. Dor: https://doi.org/10.18601/01229893.n38.04
} 
vigente y a la jurisprudencia reciente sobre el particular, para proponer algunas líneas de acción de lege ferenda, para definir el alcance de la consulta y una propuesta interpretativa, ya de lege lata, en clave constitucional sobre la figura en cuestión. La tesis que pretende sostenerse es que a partir del marco jurídico vigente es posible predicar la existencia de la figura de forma particular para ciertos reglamentos, tal y como se establece en normas especiales, pero también en forma genérica a la luz de lo dispuesto en el artículo 8.8 CPACA. Sin embargo, debido a la forma imprecisa en que quedó redactada la figura sería deseable que el legislador se ocupe de desarrollarla o que el Consejo de Estado en su interpretación unifique sus implicaciones. Mientras ello ocurre, es posible, a la luz de una interpretación en clave constitucional de la misma, proponer ciertos parámetros para definir su alcance en la actualidad.

PALABRAS CLAVE

Reglamento, consulta pública, participación, procedimiento administrativo.

\section{ABSTRACT}

The present article addresses the figure of previous and public consultation in administrative rulemaking in the Colombian legal system. Doing that, the article uses a comparative law, a systematic interpretation and a jurisprudential approach to propose some de lege ferenda and de lege data interpretation on the figure. The thesis aims to hold is that from the existing legal framework it is possible to preach the existence of the figure form certain regulations, but also in a generic form in the light of the provisions of Article 8.8 of CPACA. However, due to the imprecise way the consultation was regulated there will be desirable that the legislative branch develop it in more detail manner in the near future or the Consejo de Estado as the Colombian supreme court on this particular issue defining in a unified way the scope of the public consultation. While this occurs, this paper propose a constitutional interpretation of the institution.

KEYWORDS

Regulation, public consultation, participation, administrative procedure.

\section{INTRODUCCIÓN}

Debido tal vez a la experiencia política iberoamericana, en el idioma castellano se encuentra acuñado el término "alcaldada" para hacer referencia a la "acción arbitraria o inconsiderada que ejecuta un alcalde o cualquier 
persona que abusa de la autoridad". Esta práctica, que no solo es predicable de las autoridades locales, sino de toda autoridad pública, no ha dejado de ser común en el devenir político y administrativo en nuestro Estado colombiano. Así, la sucesión inmediata de reglamentos que pretenden regular la integralidad de un régimen jurídico en un corto período, la expedición de otros sin la debida planeación y sustento técnico, que son derogados días después de su expedición, o la suspensión provisional de los mismos por la jurisdicción contencioso administrativa debido a su flagrante vulneración al ordenamiento jurídico, siguen siendo prueba de la necesidad de diseñar e implementar medidas que procuren limitar la arbitrariedad en el ejercicio de competencias públicas, así como la transparencia en dichas labores.

En este orden de ideas, la transparencia del sistema regulatorio se concibe, cada vez más, como un elemento fundamental para crear un entorno normativo estable y accesible, que promueva la competencia, la participación y la inversión, y que ayude a protegerse de influencias negativas de grupos de interés. En este contexto, se afirma que la transparencia refuerza la legitimidad, la equidad, la predictibilidad y la eficacia de los procesos regulatorios. Estas ideas han conducido a que se hable cada vez con mayor frecuencia de la necesidad de unas mejores políticas regulatorias, que contribuyan a una idea de "buena gobernanza" 2

Para ello se han venido diseñando una serie de prácticas para llevar a cabo dicho propósito, entre las que se encuentran procesos estandarizados para elaborar y modificar regulaciones, realización de hojas de ruta previas a la expedición de la regulación, la aplicación de estudios de impacto de las medidas regulatorias ex ante y ex post, entre otras ${ }^{3}$. Dentro de estos mecanismos se ha propuesto igualmente la implementación de procedimientos de consulta pública, previos a la expedición de regulaciones, por parte de las autoridades administrativas con los sujetos destinatarios e interesados en la regulación. Al respecto se ha sostenido que la consulta eficaz es una consideración clave para garantizar que los intereses de ciudadanos y empresas se tomen en cuenta al desarrollar y diseñar regulaciones. En este sentido, la consulta pública mejora la efectividad de las regulaciones mediante el uso de la información que entidades y personas reguladas aportan sobre sus probables impactos, y por tanto es oportuno poner a consideración los problemas y las posibles deficiencias, aumentando el compromiso de los interesados y promoviendo una mayor probabilidad de cumplimiento ${ }^{4}$.

1 Real Academia de la Lengua. Diccionario de la Lengua Española, s.v. "alcaldada", disponible en: http://dle.rae.es/?id=1bNb7jq

2 OCDE (2013). Colombia: Implementing good governance. Paris: OECD Publishing, 2013, 95.

3 XanthaKi, H. (2014). Drafting Legislation: Art and Technology of Rules for Regulation. London: Hart Publishing.

4 OCDE, Estudio de la OCDE sobre la política regulatoria en Colombia: más allá de la simplificación administrativa. Paris, OECD Publishing, 2014, 91. 
En este orden de ideas, el presente escrito se dirige a analizar la figura de la consulta pública en el sistema jurídico colombiano, en particular, aquella que tiene lugar en un momento previo a la expedición de reglamentos por parte de autoridades administrativas. Para estos efectos, el presente escrito se estructura en cuatro apartados. En un primer lugar, se analizará desde el derecho comparado cómo ha sido regulada la figura de la consulta pública de proyectos de reglamentos, tanto en sistemas jurídicos nacionales como en algunas organizaciones internacionales. En un segundo apartado, se analizará el marco jurídico vigente en Colombia relativo a dicha figura, revisando para ello el nivel constitucional, legal y reglamentario vigente. En un tercer apartado, se estudiará la incipiente jurisprudencia del Consejo de Estado en la que ha abordado la figura y sus efectos en el sistema jurídico. Y por último, en un cuarto apartado, se hará una evaluación del marco antes descrito, planteando unas propuestas de lege ferenda y de lege lata, presentando, en este último sentido, una propuesta de interpretación de la consulta pública a la luz del marco normativo vigente.

Antes de continuar, resulta conveniente realizar al menos dos precisiones sobre el objeto de estudio. Por un lado, con el término reglamentos se hace mención a los actos administrativos de contenido general y abstracto, con vocación de permanencia y expedidos con fundamento en el ejercicio de la potestad reglamentaria ${ }^{5}$, siendo un ejemplo típico de ellos, pero no exclusivo, los decretos reglamentarios expedidos por el Gobierno Nacional con fundamento en el artículo 189.11 constitucional. Por otro lado, se precisa que el objeto de análisis del artículo se distingue del derecho fundamental a la consulta previa de las comunidades étnicamente diferenciadas, en los términos del Convenio 169 de la orT, en tanto dicha consulta dispone de un fundamento teleológico y normativo diferente al propuesto en el presente artículo.

Hechas estas precisiones, valga destacar que el objeto de estudio identificado reviste la mayor pertinencia y oportunidad, teniendo en cuenta que la actividad reglamentaria ha adquirido una inusitada trascendencia en las últimas décadas, tanto a nivel mundial como nacional. Por un lado, ya que es a través de normas infra-legales que se han regulado trascendentales ámbitos de la vida humana como lo son el medio ambiente, la seguridad alimentaria, la seguridad pública, la competencia, la salud pública, la regulación de los mercados financieros, las migraciones, entre otras. Y por otro, en tanto son este tipo de reglamentos los que tienen influencia directa sobre la vida cotidiana de las personas, determinando, en primera medida, el alcance pero también las restricciones al ejercicio de los derechos de los que son titulares. Por lo tanto, por el uso cada vez más frecuente y con mayor impacto sobre

5 Quinche Ramírez, M. y Urrego Ortiz, F. Los decretos presidenciales en el sistema normativo. Bogotá, Doctrina y Ley, 2011, 198. 
la vida de las personas que tiene en la actualidad la actividad reglamentaria, preguntarnos por cómo incrementar la transparencia, legitimidad y eficacia de su expedición, a través de la participación, adquiere la mayor relevancia y pertinencia.

Por otro lado, este artículo pretende contribuir a la construcción de una dogmática en torno al procedimiento administrativo; en este caso, tendiente a la producción normativa por parte de la administración, asunto que, como lo afirma López Medina ${ }^{6}$, ha sido un asunto relegado por la relevancia de la teoría del acto en la construcción del derecho administrativo en Colombia. Esta propuesta, que se enmarca dentro de la denominada "procesalización" de la acción administrativa, que se presenta como una estrategia para restringir la arbitrariedad de la administración y garantizar el debido proceso administrativo, claramente se adecúa a la teleología y contenido del Código de Procedimiento Administrativo y de lo Contencioso Administrativo (en adelante $\mathrm{CPACA}$ ), y se concentra, para los efectos que nos convocan, en la participación ex ante, antes que en el simple ejercicio de la autoridad, lo cual plantea perspectivas novedosas para formas más democráticas, participativas y transparentes de gestión pública y, por ende, de construcción y aplicación del derecho administrativo en Colombia.

\section{LA CONSULTA PÚBLICA DE LOS REGLAMENTOS \\ EN EL DERECHO COMPARADO}

La participación ciudadana en los espacios de toma de decisiones públicas ha sido reivindicada de tiempo atrás. Si bien podría afirmarse que es precisamente a ello a lo que apunta la democracia como sistema político ${ }^{8}$, han sido esfuerzos teóricos más recientes los que se han centrado en destacar la relevancia de la deliberación previa a la adopción de la decisión mayoritaria, hasta el punto de considerarla como un elemento definitorio de la concepción contemporánea de la democracia ${ }^{9}$. En nuestros días, estas ideas normativas de la política han adquirido una gran practicidad, hasta el punto de que desde la ciencia política y de la administración pública es cada vez más unánime la reivindicación de los mecanismos de participación ciudadana en las dis-

6 López Medina, D. Prólogo. En Peña Porras, D. I. La construcción del derecho administrativo colombiano. Bogotá, Universidad de los Andes y Temis, 2008, XVI.

7 Zárate Pérez, A. R. "La eficacia de la acción administrativa frente al fenómeno de procesalización”. En Montaña Plata, A. y Ospina Garzón, A. F., eds. La constitucionalización del derecho administrativo. Bogotá, Universidad Externado de Colombia, 2014, 572.

8 Dunn, J. Democracia, el viaje inacabado (508 a.C. a 1993). Barcelona, Tusquets, 1995.

9 Nino, C. S.. La construcción de la democracia deliberativa. Barcelona, Gedisa, 1997. 
tintas etapas de desarrollo de las políticas públicas ${ }^{10}$ y de las actuaciones gubernamentales ${ }^{11}$.

Desde esta perspectiva el derecho administrativo no ha sido la excepción. Así, Javier Barnes ${ }^{12}$, en un texto ya clásico en la literatura especializada, nos propone desde una perspectiva de derecho comparado la existencia de tres grandes generaciones del procedimiento administrativo. La primera generación, que puede ubicarse en la madurez del derecho administrativo, remite a la noción de procedimiento como instrumento o mecanismo para la correcta aplicación del derecho en manos de la administración, el cual estaba especialmente diseñado para el dictado de actos administrativos de contenido particular y concreto. En este sentido, señala el autor que el modelo utilizado en esta primera generación se asimila al proceso judicial y que su típico ejemplo lo constituye el ejercicio de la actividad de policía administrativa frente a un ciudadano al expedir un acto administrativo con contenido particular.

La segunda generación de procedimientos surge con fuerza a partir de la segunda mitad del siglo xx, y tiene por objeto la adopción de actos administrativos generales y abstractos, es decir, normas infra-legales que pueden identificarse en la doctrina con el término de reglamentos. Estos procedimientos se habrían de construir sobre la imagen del procedimiento legislativo y si bien, en su generalidad, se basan en la potestad de la administración para decidir sobre los asuntos, abogan por cierta participación de las partes interesadas con el fin de procurar transparencia en la regulación. Por último, se identifica en el texto el surgimiento de una tercera generación de procedimiento administrativo, centrada en la noción de "gobernanza", la cual, basada en un modelo de administración cooperativa o en red, determina junto con el sector privado y los grupos de interés nuevas formas de dirección y regulación, a través de mecanismos público-privados e interadministrativos.

A pesar de lo relevante de la identificación de esta nueva generación de procedimientos administrativos para el devenir del derecho administrativo valga señalar, para el asunto que pretende abordarse, que el tema de la participación en la expedición de reglamentos corresponde a lo que Barnes identifica como procedimientos administrativos de segunda y tal vez de tercera generación, los cuales tienen auge a lo largo de la segunda mitad del siglo Xx. A partir de esta ubicación en el derecho comparado, valga advertir que, según lo señalado anteriormente, el alcance pretendido con este artículo no es el de abordar en su integridad el procedimiento administrativo que puede

10 Subirats, J. "Nuevos mecanismos participativos y democracia: promesas y amenazas". En Font, J., coord. Ciudadanos y decisiones públicas. Barcelona: Ariel. 2001.

11 Callahan, K. Elements of Effective Governance: Measurement, accountability and participation. New York, Taylor \& Francis. 2007.

12 BARnes, J. "Tres generaciones de procedimiento administrativo". En Aberastury, P. y BlANKe, H.J., coords. Tendencias actuales del procedimiento administrativo en Latinoamérica y Europa. Buenos Aires, Eudeba, 2011. 
anteceder la expedición de reglamentos, sino únicamente la posibilidad de que dentro del mismo se prevea la existencia de consultas que permitan la participación de los ciudadanos y grupos de interés. Con esta advertencia, nos centraremos en esbozar la existencia de dichos mecanismos en los sistemas jurídicos de Estados Unidos y España, y posteriormente en la Unión Europea, para concluir con las recomendaciones que al respecto vienen realizando organizaciones multilaterales de cooperación como la Organización para la Cooperación y el Desarrollo Económico (OCDE).

Como bien nos los recuerda López Medina ${ }^{13}$, el derecho administrativo anglo-americano se ha caracterizado por centrarse más en el "procedimiento administrativo" que conduce a la formación de la decisión, con amplia audiencia y participación de los grupos sociales y económicos interesados, que en el acto administrativo mismo. En este sentido, y a raíz de la influencia ganada por las agencias federales tras la implementación del New Deal, el Congreso estadounidense expidió en 1946 la Ley sobre Procedimientos Administrativo (APA por sus siglas en inglés) ${ }^{14}$, como un mecanismo para controlar el funcionamiento de las mismas ${ }^{15}$. Sobre los propósitos que persigue este procedimiento de consulta se ha dicho que aumenta la calidad y legitimidad de las políticas públicas, al generar mayor información disponible e impedir que intereses particulares ejerzan una influencia indebida ${ }^{16}$. Ya frente al procedimiento de producción de normas por parte de las agencias administrativas, y a pesar de que por la generalidad de la ley se puede hablar de la existencia de distintos procedimientos, en la actualidad tiene lugar cierto protagonismo el que suele denominarse como "notice and comment", que puede traducirse como "aviso y comentario" 17 .

Este procedimiento parte de establecer al menos cinco requerimientos a las agencias, antes de expedir las regulaciones correspondientes ${ }^{18}$. En primer lugar, debe tanto notificarse a las personas potencialmente afectadas con la expedición de la nueva regulación como publicarse un aviso en el Registro Federal (Federal Register) sobre la propuesta de regulación. En segundo lugar, la agencia deberá dar a las personas interesadas la oportunidad de participar en la creación de la norma, a través del envío de información escrita, opiniones u otros argumentos. Tercero, una vez procesada la información presentada, la agencia deberá producir y publicar en el Registro Federal un

13 López MEDINA, ob. cit.

14 Administrative Procedure Act [Public Law 404 - 79th Congress], aprobada el 11 de junio de 1946 por el Congreso de los Estados Unidos de América.

$15 \mathrm{Al}$ respecto véase McNollgast, $\mathrm{H}$. The political origins of the Administrative Procedure Act. The Journal of Law, Economics \& Organization, Oxford, Oxford Journals, 1999, vol. 15.

16 Rosenbloom,D.H.Administrative law for public managers. Washington D.C.: Westview Press, 2003, 62 .

17 IBÍD., 66.

18 Ibíd. 
texto definido con su contenido y propósito. En cuarto lugar, tras la publicación deberá seguir un lapso de, al menos, treinta días antes de la entrada en vigencia de la regulación. Por último, durante ese plazo la agencia debe dar a los interesados el derecho de pedir la revisión, modificación o anulación de la regulación adoptada, y deberá considerar todos aquellos que reciba ${ }^{19}$.

De lo anterior es claro que, si bien se trata de un procedimiento flexible para la expedición de regulaciones, y que la existencia de excepciones a este son comúnmente utilizadas, sin lugar a dudas corresponde este a un esfuerzo relevante por generar y abrir espacios de participación y consulta para la expedición de reglamentos federales ${ }^{20}$. Incluso, hoy en día es cada vez más común que las agencias procuren dar cumplimiento copioso y riguroso a dicho procedimiento previendo la defensa de las mismas regulaciones ante las cortes federales y como estrategia para probar la razonabilidad de las mismas ${ }^{21}$.

En este sentido puede señalarse que el sistema estadounidense de "aviso y comentario" ha generado un proceso regulatorio abierto y accesible en el nivel federal, en tanto es un proceso abierto a todos los ciudadanos, lo que hace que se distinga de aquellos utilizados en modelos de consulta más corporativistas y también de los métodos informales que otorgan una considerable discreción para decidir a quién consultar. Estos aspectos han conducido a que a nivel comparado el modelo norteamericano sea visto como un procedimiento que respeta las buenas prácticas internacionales de transparencia ${ }^{22}$.

Por su parte, en España, la Constitución vigente desde 1978 dispone en el artículo 105.a) que la ley regulará "la audiencia de los ciudadanos, directamente o a través de las organizaciones y asociaciones reconocidas por la ley, en el procedimiento de elaboración de las disposiciones administrativas que les afecten" 23 , por lo que se ha entendido unánimemente que el principio de la participación de los ciudadanos en la elaboración de los reglamentos tiene rango constitucional ${ }^{24}$. Dicho procedimiento de elaboración de reglamentos por el Gobierno se encuentra establecido principalmente en la Ley 50/1997, más conocida como la Ley del Gobierno ${ }^{25}$. Frente a la existencia de procedimientos de consulta en dicho procedimiento debe señalarse que

19 Para profundizar al respecto véase StRAuss, Peter L. Los procedimientos de elaboración de reglamentos y disposiciones administrativas en EE.UU. En Barnes, J., ed. La transformación del procedimiento administrativo. Sevilla: Derecho Global, 2008.

20 Connelius, K. Rulemaking: How Government Agencies Write Law and Make Policy. 2. ${ }^{\text {a }}$ ed. Washington, D.C.: CQ Press, 1999.

$21 \mathrm{Al}$ respecto véanse los casos: Citizens to Preserve Overton Park Inc. v. Volpe, $401 \mathrm{US}$ 402, 415 (1971); Chamber of Commerce of US v. Osha F. 2d 464, 470 (D.C. Cir. 1980).

22 OCDE (2014), 91.

23 Constitución española, 1978.

24 SÁnchez Morón, M. Derecho administrativo: parte general, Madrid, Tecnos, 2012, 214.

25 Ley 50/1997, de 27 de noviembre, del Gobierno. BOE n. ${ }^{\circ} 285$, de 28 de noviembre de 1997, pp. 35082-35088. 
se prevén en dos distintos momentos ${ }^{26}$. Por un lado, durante la elaboración del proyecto, donde se faculta al Gobierno para que realice las consultas que considere convenientes "para garantizar el acierto y la legalidad del texto" (art. 24.1.b), y de lo cual deberá dejar constancia en el expediente (art. 24.1.f). Y por otro lado, una vez elaborado el proyecto de reglamento y que este afecte a los derechos e intereses legítimos de los ciudadanos, señala la ley que se dará audiencia a los ciudadanos afectados, durante un plazo razonable y no inferior a quince días hábiles, directamente o a través de las organizaciones y asociaciones reconocidas por la ley que los agrupen o los representen y cuyos fines guarden relación directa con el objeto de la disposición ${ }^{27}$ (art. 21.1.c). Continúa la norma señalando que la decisión sobre el procedimiento escogido para dar audiencia a los ciudadanos afectados será debidamente motivada en el expediente por el órgano que acuerde la apertura del trámite de audiencia. Asimismo, y cuando la naturaleza de la disposición lo aconseje, será sometida a información pública durante el plazo indicado ${ }^{28}$.

Como excepciones a dicha consulta se establecen dos. En primer lugar, cuando sobrevengan "graves razones de interés público", que asimismo deberán explicitarse. En segundo lugar, cuando el proyecto de reglamento se refiera a los órganos, cargos y autoridades del Gobierno, así como a las disposiciones orgánicas de la Administración General del Estado o de las organizaciones dependientes o adscritas a ella. Por último, se señala que dicho trámite podrá ser abreviado hasta el mínimo de siete días hábiles cuando razones debidamente motivadas así lo justifiquen. Salvo estas dos excepciones mencionadas, que en cualquier caso requiere que así se justifiquen previamente, el reglamento estatal aprobado omitiendo ese trámite es nulo ${ }^{29}$. Por lo tanto, si bien dicho procedimiento ha sido calificado por algunos doctrinantes como "rudimentario" 30 y que su aplicación "dista de ser satisfactoria [por estar] muy alejada de las fórmulas y del alcance de la participación que tiene lugar en otros países democráticos" ${ }^{31}$, constituye sin lugar a dudas un mecanismo de consulta pública previa y de carácter obligatorio a la expedición de reglamentos, en los términos que nos convocan.

Por otro lado, desde el año 2009 y a propuesta del presidente José Manuel Durao Barroso, la Unión Europea puso en marcha la política conocida como Smart Regulations (Normativas Inteligentes) con el fin de mejorar la calidad y la eficacia de la normatividad expedida por los órganos de la Unión para

26 SÁnchez Morón, ob. cit., 216.

27 Al respecto véanse las sentencias del Tribunal Supremo de España del 14 de febrero de 2003, el 23 y el 26 de septiembre de 2003.

28 García de Enterría, E. y Fernández, T.R. Curso de derecho administrativo, 10. a ed., Madrid, Civitas, 2000, p. 192.

29 SÁNCHEZ Morón, ob. cit., 214.

30 BARNES, ob. cit., 67.

31 SÁnChEZ MoRón, ob. cit., 214. 
responder a los problemas coyunturales que la aquejaban para entonces. Dentro de esta política cabe resaltar, para los efectos de este artículo, el lugar central que en ella ocupan los destinatarios o "usuarios finales" de las normas. En este sentido se pronunció expresamente el presidente Barroso: "[L]a revitalización de los vínculos entre los pueblos de Europa y la UE hará a ésta más legítima y más efectiva. Dar la capacidad a los ciudadanos de participar en las decisiones que afectarán a sus vidas, garantizado entre otras cosas la transparencia sobre cómo se toman, ayudará a lograr estos objetivos" ${ }^{32}$.

En el marco de esta política, los "usuarios finales" de la normatividad son vistos desde una posición privilegiada para facilitar una información pertinente y actualizada que podría mejorar la calidad de los datos en los que se basan las decisiones. Para ello, la política de "Normatividad inteligente" precisa tres mecanismos fundamentales ${ }^{33}$, a saber: (i) la existencia de oportunidades para la participación de los destinatarios de las normas; (ii) la existencia de incentivos y mecanismos de gobierno de probada solidez que garanticen que sus contribuciones se tomen debidamente en cuenta, y (iii) que se lleve un seguimiento cuantitativo y cualitativo de la marcha del proceso y que se comunique a los usuarios finales de un modo sistemático. Estos mecanismos, si bien tienen incidencia en todos los órganos de la Unión que participan en el procedimiento legislativo, como el Parlamento y el Consejo, tienen un efecto directo en la Comisión, ya que es esta quien se reserva la gran generalidad de iniciativas legislativas dentro de la Unión ${ }^{34}$.

En la actualidad, y como desarrollo de dicha política, existen al menos tres mecanismos desarrollados por la Comisión Europea para mejorar los procedimientos de consulta pública de las normas expedidas ${ }^{35}$. En primer lugar, a través de la participación de la hoja de ruta y creación de la normatividad. En ella se publica la justificación y documentos técnicos que podrían originar la expedición de una normativa, exponiendo su contexto, definición del problema, objetivos, alternativas, un análisis inicial de impacto. Estos documentos luego son sujetos a consultas con los interesados, sean organizaciones o ciudadanos, que se harán públicamente a través de la página web, por periodos variables y donde la Comisión tiene el deber de contestar a los aportes enviados. Otra forma de participación tendrá lugar a través de paneles con expertos en la materia donde se discuten los fundamentos de las normatividades.

En segundo lugar, dicha participación viene teniendo lugar a través de los análisis de impacto de los proyectos normativos. Ellos suelen hacerse

32 Citado por Unión Europea (2010). Normativa inteligente: documento de síntesis. Bruselas, Unión Europea.

33 Ibíd., 16.

34 SÁnchez, V. M. Derecho de la Unión Europea, Madrid, Huygens, 2012, 154.

35 Ibíd. 
por consulta pública sobre los impactos que pueden suponer los proyectos de regulación por parte de grupos de interés que puedan verse afectados con la expedición de dichas normas. Estas suelen hacerse vía correo o vía portal web, durante un periodo aproximado de dos meses y donde se reciben, compilan, publican y responden las contribuciones realizadas. En tercer y último lugar, existe también la posibilidad de proponer iniciativas de creación o modificación de normatividades existentes. En ella se reciben y publican las iniciativas que se tengan sobre nuevas regulaciones o reformas de las regulaciones existentes, las cuales son igualmente publicadas para recibir retroalimentaciones de los ciudadanos y grupos de interés. Este mecanismo se encuentra actualmente en construcción y espera constituirse en un punto central para la creación de nuevas regulaciones en un futuro cercano ${ }^{36}$.

Por último, organismos multilaterales como el Banco Mundial ${ }^{37}$ y la OCDE han abogado en las últimas décadas por la necesidad de introducir mejoras en las políticas normativas dentro de los Estados parte. En este sentido, la OCDE ha venido insistiendo en la necesidad de adoptar por parte de los Estados miembros y afiliados ciertos estándares que conduzcan a una adecuada gobernanza pública. Dentro de dichos estándares consta que para una adecuada política regulatoria deben establecerse procedimientos únicos y estandarizados que ayuden a la preparación, socialización y discusión de los proyectos de regulación preparados. Así, en 2012, la Recomendación del Consejo de la OCDE sobre política y gobernanza regulatoria sugirió a los gobiernos "establecer mecanismos e instituciones para supervisar activamente los procedimientos y objetivos de la política regulatoria, apoyarla e implementarla, y por consecuencia fomentar la calidad de la regulación" 38 .

Para ello, la OCDE ha encontrado que adoptar procedimientos comunes y publicar documentos guía es particularmente importante para promover un constante compromiso con la consulta pública en el seno de la administración. Las guías para la consulta tienen dos fines: en primer lugar, expresan con claridad el compromiso de política pública del Gobierno de requerir a los funcionarios tomar en cuenta a los ciudadanos. En segundo lugar, brindan una valiosa orientación técnica para los funcionarios sobre la manera de diseñar una consulta pública eficaz y de integrar las opiniones del público ${ }^{39}$.

Incluso dentro del proceso de ingreso de Colombia a la OCDE, dicho organismo de cooperación ha tenido oportunidad de indagar y conceptuar sobre los mecanismos de participación que se disponen previo a la expedición de

36 XanthaKi, H. "EU Legislative Quality post-Lisbon: The challeges of Smart Regulation". Statute Law Review, (2014) 35, 66 ss.

37 Islam, R. Do more transparent Governemnt Govern Better? Washington D.C., Banco Mundial, 2003.

38 OECD, Recomendación del Consejo sobre Política y Gobernanza Regulatoria. Paris, OCDE, 2012, 45.

39 Ibíd. 
reglamentos. Al respecto ha señalado el grupo de expertos de la OCDE que en Colombia no existe homogeneidad en la manera en que se realizan las consultas públicas, lo cual contrasta marcadamente con la experiencia internacional, y por tanto ha recomendado expresamente al país "[d]esarrollar e implementar estándares obligatorios sobre el uso de la consulta pública como medio para hacer partícipes a los ciudadanos, las empresas y la sociedad civil en el proceso regulatorio y obtener mejores resultados de política pública" ${ }^{40}$.

\section{LA CONSULTA PÚBLICA EN EL MARCO \\ NORMATIVO VIGENTE EN COLOMBIA}

Establecidos algunos estándares en el derecho comparado en relación con la participación para la preparación y expedición de reglamentos, corresponde ahora analizar el marco normativo vigente colombiano relativo a dicho asunto. En este sentido, es necesario iniciar con el análisis del parámetro constitucional vigente; para lo cual se hace necesario distinguir entre el canon constitucional vigente y las normas que hacen parte del bloque de constitucionalidad en sentido estricto. Al respecto debemos afirmar que si bien no existe una disposición expresa que disponga el deber de las autoridades administrativa de abrir espacios de participación anteriores a la expedición de actos administrativos de contenido general y abstracto, sí existen valores y principios constitucionales que guardan relación con el particular.

Así, desde el mismo Preámbulo de la Constitución se afirma que el pueblo colombiano se otorga un marco participativo, el cual deriva de definir al Estado colombiano, en el artículo siguiente, como una República "participativa", entre otros adjetivos. En esta misma línea, el artículo 2. ${ }^{\circ}$ de la Constitución vigente, al señalar los fines esenciales del Estado colombiano, dispone como uno de ellos el de "facilitar la participación de todos en las decisiones que los afectan y en la vida económica, política, administrativa y cultural de la Nación" ${ }^{41}$. Además el artículo 40 ibídem señala igualmente que "Todo ciudadano tiene derecho a participar en la conformación, ejercicio y control del poder político"42.

Sobre los ámbitos del derecho a la participación ha señalado la Corte Constitucional, interpretando dicha disposición constitucional, que en virtud de ella el ciudadano interviene en su conformación, al ordenar, estructurar e integrar el poder político; en el ejercicio, al practicar, desplegar o manifestar la titularidad del poder político; y en el control, al vigilar, explorar y examinar la gestión de los órganos que expresan institucionalmente el poder político.

40 OCDE, Estudio de la OCDE sobre la política regulatoria en Colombia: más allá de la simplificación administrativa. Paris, OECD, 2014, 24.

41 Colombia. Constitución Política de Colombia, art. 2..

42 Ibíd., art. 40. 
Y en particular sobre la dimensión de la participación como control dispuso el alto tribunal que "de la Constitución se sigue [también] el derecho de los ciudadanos a contar con mecanismos que hagan posible el diálogo con las autoridades públicas así como el control de la gestión que desarrollan y sus resultados (arts. 40, 103 y 270) -el derecho a la democracia 'como control'-"'43.

De igual forma, ha señalado la jurisprudencia constitucional, de manera reiterada, que el principio participativo que inspira la Constitución vigente tiene dos características fundamentales, a saber, su carácter universal y expandible ${ }^{44}$. En relación con el carácter universal, se ha señalado que la participación compromete variados escenarios, procesos y lugares tanto públicos como privados, y también que la noción de política que lo sustenta se nutre de todo lo que vitalmente pueda interesar a la persona, a la comunidad y al Estado, y sea por tanto susceptible de afectar la distribución, control y asignación del poder social. Por su parte, el carácter expansivo se refiere a que este ha de ampliarse progresivamente, conquistando nuevos ámbitos y profundizando permanentemente su vigencia.

Por otra parte, el artículo 209 constitucional dispone que la función administrativa está al servicio de los intereses generales y se desarrolla con fundamento en los principios de igualdad, moralidad, eficacia, economía, celeridad, imparcialidad y publicidad, los cuales guardan una directa relación con la finalidad propuesta por los procedimientos consultivos. Continuando con el análisis del marco constitucional vigente, encontramos que en el denominado bloque de constitucionalidad ${ }^{45}$ no se encuentran normas específicas que aborden la temática referida, salvo la consagración del derecho político de "participar en la dirección de los asuntos públicos, directamente o por medio de representantes libremente elegidos", que consta con igual contenido en los artículos 25 literal a) y 23 numeral 1 literal a) del Pacto Internacional de Derechos Civiles y Políticos y de la Convención Americana de Derechos Humanos, respectivamente.

Así, de lo dicho hasta este punto puede afirmarse que del canon constitucional positivo se desprende que la participación no solo es un valor sino un derecho constitucional, que por un lado no se agota en el ámbito político sino que incluye otros ámbitos como la vida "administrativa" de los ciudadanos, y por otro incluye la dimensión de la participación como control, la cual exige que existan mecanismos de diálogo, incluso directo, con las autoridades políticas y administrativas antes de la adopción de decisiones

43 Colombia. Corte Constitucional, Sentencia C-150 de 2015, M.P.: Mauricio Gonzalez Cuervo.

44 Colombia. Corte Constitucional, Sentencia C-089 de 1994, M.P.: Eduardo Cifuentes Muñoz.

45 Entre otras, véase Corte Constitucional, Sentencia C-225 de 1995, M.P.: Alejandro Martínez Caballero. 
que los afecten. Además, con fundamento en la doctrina constitucional dicho derecho deberá entenderse como un mandato de optimización a la luz de los criterios de universalidad y expansibilidad de la participación. Por último, debe tenerse en cuenta que toda función administrativa, incluyendo la expedición de reglamentos, debe estar inspirada en los principios de moralidad, imparcialidad y publicidad, entre otros, por expreso mandato constitucional.

Descendiendo ya al nivel legal vigente, encontramos un importante precedente al respecto pero que fue bastante fugaz en nuestro ordenamiento jurídico ${ }^{46}$. En vigencia encontramos solo dos disposiciones que tienen relevancia para el tema que nos convoca. En primer lugar está lo dispuesto por el artículo $7 .^{\circ}$ de la Ley 1314 de $2009^{[47]}$, el cual establece algunos criterios a los que debe sujetarse la regulación relativa a normas de contabilidad e información financiera, y al hacerlo ordena a los ministerios correspondientes realizar una serie de consultas públicas antes de la adopción de la regulación particular. Para ello dispone que a los proyectos deberá aplicárseles un análisis de impacto, tras el cual se le podrán hacer observaciones y recomendaciones al proyecto inicial por parte de las entidades públicas interesadas en el tema. Luego de ello dispone la ley que deberá elaborarse un texto definitivo del proyecto, para el cual "se analizarán y acogerán, cuando resulta pertinente, las observaciones realizadas durante la etapa de exposición pública de los proyectos" ${ }^{48}$, sin que se haga mayor claridad al respecto sobre ello. Esta, si bien es una norma dirigida a regular la expedición de un campo regulatorio muy preciso, resulta relevante en tanto es la primera disposición legal vigente que regula algún tipo de consulta pública previa a la expedición de un reglamento. Además, puede señalarse que el Consejo Técnico de Contaduría Pública, como organismo de normalización técnica de normas contables, viene dando aplicación a lo antes dispuesto a través de su portal web por medio de "discusiones públicas" a las cuales somete sus proyectos de regulación en la materia.

La segunda disposición legal al respecto es más general y se encuentra dentro de la Ley 1437 de 2011, "Por medio de la cual se expide el Código

46 El Decreto Ley 266 de 2000, expedido por el Gobierno de Andrés Pastrana Arango, reguló en los artículos 31 a 33 una suerte de procedimiento para la expedición de ciertas regulaciones, tanto en el orden nacional como en el departamental, distrital y municipal. Dentro de este se exigía la publicidad de los proyectos de los mismos (art. 31), en ellos debía establecerse un plazo para la recepción de observaciones, sugerencias o propuestas alternativas que no podría sea inferior a una semana (art. 32), y en la motivación del reglamento adoptado se debía señalar la motivación sobre la aceptación o rechazo de las observaciones formuladas (art. 33). Sin embargo, dicho decreto solo estuvo vigente un poco más de seis meses, debido a que la Corte Constitucional, mediante Sentencia C-1316 de 2000, lo declaró inexequible en su integridad debido a la imprecisión de las facultades extraordinarias concedidas por la Ley 573 de 2000, las cuales servían de fundamento a la expedición del decreto ley mencionado.

47 Colombia. Congreso de la República. Ley 1314 del 13 de julio de 2009.

48 Ibíd. 
de Procedimiento Administrativo y de lo Contencioso Administrativo" 49 (en adelante CPACA), particularmente en su artículo $8 .^{\circ}$, al regular el deber de información al público por parte de la autoridades administrativas. En relación con ello, señala expresamente la norma en mención:

Las autoridades deberán mantener a disposición de toda persona información completa y actualizada, en el sitio de atención y en la página electrónica, y suministrarla a través de los medios impresos y electrónicos de que disponga, y por medio telefónico o por correo, sobre los siguientes aspectos: $[\ldots]$

8. Los proyectos específicos de regulación y la información en que se fundamenten, con el objeto de recibir opiniones, sugerencias o propuestas alternativas. Para el efecto, deberán señalar el plazo dentro del cual se podrán presentar observaciones, de las cuales se dejará registro público. En todo caso la autoridad adoptará autónomamente la decisión que a su juicio sirva mejor el interés general.

De la lectura de dicha disposición legal pueden abstraerse los siguientes enunciados normativos: (i) se consagra un deber legal predicable de todas las autoridades, en los términos del artículo 2. ${ }^{\circ}$ CPACA y, por ende, un derecho subjetivo de toda persona; (ii) el contenido del derecho se refiere a mantener a disposición de toda persona los proyectos específicos de regulación y la información en que se fundamenten en el sitio de atención y en la página electrónica, y suministrarla, en caso de requerirse, a través de medios impresos, electrónicos, telefónicos o por correo; (iii) junto con la información anterior, deberá señalarse el plazo dentro del cual se podrán presentar observaciones, a saber, opiniones, sugerencias o propuestas alternativas, de las cuales se dejará registro, y (iv) tras la publicación y consulta, la autoridad adoptará la decisión atendiendo al interés general.

Sobre la finalidad de dicha norma ha señalado la doctrina especializada que "[e]ste numeral busca incentivar la participación ciudadana respecto de los proyectos de reglamentación, de manera que tanto los interesados como los gremios, sindicatos, centros de investigación, etc., y las personas en general, puedan opinar y proponer cambios respecto de los proyectos que se publiquen" ${ }^{50}$. Por su parte, sobre el artículo $8 .^{\circ}$ se ha dicho que él "concreta mediante obligaciones específicas los principios de publicidad, de transparencia y de participación" ${ }^{51}$. Por último, Zárate Pérez ha considerado que esta norma se dirige a satisfacer las obligaciones derivadas de la nueva concepción del principio democrático, en tanto pretende asegurar una mayor

49 Colombia. Congreso de la República. Ley 1437 del 18 de enero de 2011.

50 Arboleda Perdomo, E. J. Comentarios al Nuevo Código de Procedimiento Administrativo y de lo Contencioso Administrativo. Bogotá, Legis, 2013, 27.

51 Benavides, J. L., ed. Código de Procedimiento Administrativo y de lo Contencioso Administrativo, Bogotá, Universidad Externado de Colombia, 2013, 81. 
participación de los ciudadanos en la toma de decisiones al interior de las administraciones ${ }^{52}$.

A pesar de que, como se dijo, dicha disposición establece una clara obligación legal, es igualmente cierto que sobre su alcance se pueden plantear ciertos interrogantes. En primer lugar se encuentra el significado en el sistema jurídico colombiano del término "regulación". En segundo lugar, la norma dispone de un amplio margen de discreción respecto a los medios, el tiempo, las respuestas y la información publicable. En tercer lugar, se encuentra la cuestión relativa al efecto de pretermitir dicha publicidad y consulta, y a cuál es la instancia que se encarga de su verificación, entre otras. Debido a que en el nivel legal no se encuentran respuestas expresas a dichos interrogantes, nos corresponde ahora revisar el nivel reglamentario, para identificar las disposiciones que guarden relación con el deber de consultar públicamente los proyectos de reglamentos antes de su expedición. Realizada dicha revisión se obtiene como resultado la identificación de tres cuerpos normativos relacionados con el tema, dos de ellos que precisan el deber de consulta para ciertos tipos particulares de reglamentos, y uno que se refiere a dicha consulta de forma genérica.

Siguiendo al orden cronológico en su expedición, encontramos en primer lugar el Decreto 2696 de 2004 ${ }^{[53]}$, en el cual se definen las reglas mínimas para garantizar la divulgación y la participación en las actuaciones de las comisiones de regulación. Dicho decreto determina en su artículo 9. ${ }^{\circ}$ que las comisiones deberán hacer públicos, en su página web y con antelación no inferior a treinta días a la fecha de su expedición, todos los proyectos de resolución de carácter general que pretendan adoptar. Además, dicha publicación deberá incluir el término para la recepción de las observaciones, reparos y sugerencias, que no podrá ser inferior a diez días hábiles. Por último, señala el

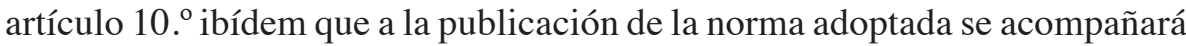
un documento que contenga las razones por las cuales se aceptan o se rechazan las propuestas formuladas. Si la regulación adoptada se refiere a asuntos tarifarios, la norma prevé, además de lo anterior, la posibilidad de celebrar consultas públicas con los usuarios y sus representantes para discutir dichos proyectos. Tal y como se señaló anteriormente, este decreto es un ejemplo de regulación de una consulta pública que se circunscribe exclusivamente a los proyectos de regulación de carácter general de la comisiones de regulación.

En segundo lugar, encontramos el Decreto 4003 de 2004 ${ }^{[54]}$, "Por el cual se establece el procedimiento administrativo para la elaboración, adopción y aplicación de reglamentos técnicos, medidas sanitarias y fitosanitarias en el ámbito agroalimentario". Dicho decreto, que fue reproducido mediante

53 Decreto 2696 del 24 de agosto de 2004.

54 Decreto 4003 del 30 de noviembre de 2004. 
Decreto Único 1071 de $2015^{[55]}$, dispone en su artículo 2.13.2.4.2 que todo proyecto de reglamento técnico, medida sanitaria o fitosanitaria elaborado por la respectiva entidad competente, deberá ser publicado en el medio de difusión de mayor cubrimiento de la respectiva entidad para dar cumplimiento a lo previsto en el CPACA, y cuando afecte el comercio internacional deberá ser notificado a través del punto de contacto de Colombia ante la OMC, CAN, G3 o cualquier otra entidad de conformidad con los acuerdos que Colombia suscriba con otros países, con el fin de recibir comentarios u observaciones.

Sobre la publicación y notificación del proyecto, dispone el mencionado decreto que deberá incluir la información para la recepción de observaciones o comentarios. Por su parte, frente a los plazos de la consulta, señala que para recibir observaciones o comentarios en los eventos de que afecten al comercio internacional, se establece un plazo no menor a noventa días calendario para los reglamentos técnicos y no menor a sesenta días calendario para las medidas sanitarias o fitosanitarias. Cuando se reciban observaciones o comentarios a través del punto de contacto, este deberá enviarlos a la entidad competente en un plazo no mayor a diez días y la entidad, señala la norma, ampliará la información en la medida que sea posible, en un plazo no mayor de noventa días calendario. Tal y como se señaló anteriormente, este decreto es un segundo ejemplo de regulación de una consulta pública que se circunscribe exclusivamente a ciertos proyectos de regulación, a saber, reglamentos técnicos, medidas sanitarias o fitosanitarias.

En tercer y último lugar, encontramos los decretos que establecen directrices de técnica normativa en el ejercicio de la potestad reglamentaria. La primera norma de este tipo la constituyó el Decreto 1345 de $2010^{[56]}$ que a la luz de lo allí dispuesto tuvo como finalidad racionalizar la expedición de decretos y resoluciones, dotar de seguridad jurídica a los destinatarios, evitar la dispersión y proliferación normativa y optimizar los recursos físicos y humanos utilizados para esta actividad. Para ello, además de adoptar directrices de técnica normativa, se adoptó un Manual para la Elaboración de Texto que debe aplicarse a todo proyecto de reglamento. Posteriormente, en el año 2015, se expidió el Decreto 1609 que modificó el Decreto 1345 e incorporó su contenido al Decreto Único del sector de la Presidencia. Si bien este decreto adicionó algunas directrices a las antes existentes, para los efectos de este escrito no supuso cambios relevantes a las disposiciones que ya estaban vigentes desde 2010. Así, en relación a los espacios de participación previos a la expedición de reglamentos, dispone el Decreto 1609 de $2015^{[57]}$ : 
Artículo 2.1.2.1.13. Deber de consultar. Cuando la Constitución y la ley así lo ordenen, deberán realizarse las consultas en ellas señaladas, caso en el cual a la memoria justificativa deberá anexarse la constancia que acredite que se ha cumplido dicho trámite.

Artículo 2.1.2.1.14. Publicidad. Cuando de conformidad con la ley, deba someterse a consideración del público la información sobre proyectos específicos de regulación antes de su expedición, a la memoria justificativa se anexará también la constancia del cumplimiento de esa obligación y se incluirá el resultado de la evaluación de las observaciones ciudadanas que se hubieren presentado.

Sin perjuicio del uso de los demás medios que se consideren necesarios para dar a conocer el proyecto a los sectores interesados, este deberá estar disponible en la sede electrónica de la respectiva entidad.

De la lectura de ambas disposiciones pueden abstraerse los siguientes enunciados normativos: (i) ambas disposiciones condicionan sus efectos a la existencia de una ley que así lo señale, la cual, a la luz de lo antes señalado y para los efectos de este escrito, la constituye el artículo 8.8 CPACA y por tanto surtirá efectos para "proyectos específicos de regulación” cuya producción no esté regulada en una normas especial; (ii) ambas disposiciones señalan que para dichas normas deberá dejarse constancia que acredite el proceso de consulta realizado en la memoria justificativa; (iii) en dicha memoria justificativa se incluirá igualmente la evaluación de las observaciones ciudadanas que se hubieran presentado, señalando su adopción o no y las razones para ello; y, (iv) dicha divulgación deberá hacerse, al menos, en la sede electrónica de la entidad que expedirá la norma, sin perjuicio de que se puedan utilizar otros medios adicionales.

Por otro lado, y si bien en rigor jurídico los documentos expedidos por el Consejo Nacional de Política Económica y Social (CONPES) no tienen naturaleza reglamentaria, como regla general, sino más de recomendaciones técnicas o documentos de política pública, en alguno de ellos encontramos aspectos relevantes para la materia en cuestión. Así, el Documento CONPES 3816, denominado "Mejora Normativa: Análisis de Impacto" 58 , aprobado el 2 de octubre de 2014, se planteó como objeto principal el de establecer las bases para institucionalizar el análisis de impacto normativo en la etapa temprana del proceso de emisión de la normatividad desde la rama ejecutiva, como herramienta dirigida a fortalecer la confianza, efectividad y transparencia de la normatividad, en el mediano y largo plazo ${ }^{59}$.

58 Documento CONPES 3816: Mejora Normativa: Análisis de Impacto, disponible en: https:// colaboracion.dnp.gov.co/CDT/Conpes/Econ\%C3\%B3micos/3816.pdf

59 Ibíd., 4. 
Para ello, el documento parte de un claro diagnóstico en el tema que nos ocupa al señalar que los mecanismos de consulta pública contemplados en la producción normativa son débilmente difundidos, poco efectivos y considerablemente heterogéneos. En este punto, si bien reconoce la existente de algunos mecanismos de consulta como los anteriormente referidos, señala que estos se vienen aplicando de manera "descoordinada y dispersa", lo cual genera un "desestímulo a la participación, además de minar la transparencia del proceso de producción normativa" ${ }^{\circ 0}$.

A partir de este diagnóstico se identifica como una de sus posibles causas el que las prácticas de consulta y transparencia asociadas al proceso de producción normativa son insuficientes para garantizar su calidad. En este punto, destaca el documento que las consultas, cuando se adelantan, tienden a realizarse de un modo general, principalmente a través de páginas web o de espacios de diálogo, sin tomar en cuenta las diferencias de la población en términos de acceso, características poblacionales y diversidad territorial. Aunado a esto, señala el CONPES que los procesos de consulta tienden a limitarse a la recepción de comentarios y propuestas, siendo poco claros en términos de la forma en la que se desarrollarán, la manera en la que se utilizarán los insumos generados, la forma como se responderá frente a los mismos y la medida en la que estos insumos podrán, o no, ser incluidos en la norma ${ }^{61}$. Por lo tanto, concluye el Documento CONPES 3816 estableciendo como recomendación el que se solicite a las entidades competentes adelantar las acciones necesarias para facilitar la incorporación del análisis de impacto normativo que incluya los criterios de consulta pública en el proceso de emisión normativa de la rama ejecutiva de orden nacional.

\section{LA CONSULTA PÚBLICA EN LA MÁS RECIENTE JURISPRUDENCIA DEL CONSEJO DE ESTADO}

Una vez analizado el marco normativo vigente para la consulta pública, corresponde ahora estudiar cómo el Consejo de Estado, como juez natural de dicha figura, ha venido pronunciándose sobre el asunto a partir de un incipiente grupo de demandas que pretenden atacar la validez de algunos reglamentos y regulaciones, al sostener, entre otros argumentos, que se ha pretermitido la instancia de publicación y consulta como la prevista en el artículo 8.8 CPACA y algunas otras normas reglamentarias que lo refuerzan.

Por las restricciones propias de este artículo y el carácter novedoso del asunto en sede jurisdiccional, se ha optado metodológicamente por rastrear los pronunciamientos que al respecto han proferido las distintas secciones del Consejo de Estado sobre el particular en el año en curso, sin distinguir, a

60 Ibíd., 16.

61 Ibíd. 
pesar de sus diferencias obvias, entre sentencia y autos con el fin exclusivo de ahondar en las posiciones iniciales que se han venido exponiendo sobre el particular. Hecho este rastreo, se han identificado al menos tres providencias proferidas durante el año en curso que han abordado el asunto, todas ellas en sede del medio de control de nulidad, siendo dos de ellas sentencias proferidas por las secciones Primera y Quinta, y un auto proferido dentro de la Sección Cuarta. A continuación se expondrán, para cada una de estas providencias, los hechos que le dieron origen, las consideraciones y la decisión adoptada por la sala de la sección correspondiente. Posterior a ello, se intentará hacer un balance de las mismas y proponer las perspectivas al respecto.

\subsection{Sentencia del 4 de febrero de 2016, proceso con radicado 11001-0324-000-2010-00329-00, C.P.: Guillermo Vargas Ayala, Sección Primera del Consejo de Estado}

En esta oportunidad se demanda a través de la acción pública de nulidad la Resolución 1057 del 23 de marzo de 2010, "Por la cual se establece el reglamento técnico sobre los requisitos sanitarios que debe cumplir la miel de abejas para consumo humano", expedida por el Ministerio de la Protección Social de entonces. Entre otros cargos, sostuvo el actor que la resolución acusada no consultó a la comunidad afectada por el impacto social y económico de su decisión, contraviniendo lo dispuesto por el Decreto 4003 de 2004 y la Resolución 3742 de 2001 de la Superintendencia de Industria y Comercio.

Definida la naturaleza de reglamento técnico de la norma demandada, frente al cargo destacado considera la Sala que el derecho a la participación de los afectados es especialmente relevante en el ámbito de los procedimientos administrativos de expedición de regulaciones, en tanto sus insumos son importantes para la calidad de la regulación, su impacto y su eficaz implementación, enriqueciendo así el proceso de construcción de las regulaciones estatales. De igual forma se destaca que dicha participación promueve valores de relevancia constitucional, entre los que se destacan la imparcialidad, eficiencia, pluralismo y legitimidad de la decisión adoptada.

Posterior a ello encuentra la Sala que si bien la resolución demandada estuvo publicada en la página web del Ministerio por el término de un mes, no se procedió a la identificación de los grupos potencialmente afectados con el reglamento, frente a lo cual señala expresamente:

Para alcanzar el propósito de la participación en esta materia, esto es, que la Administración cuente con elementos de juicio y argumentos pertinentes que permitan adoptar una determinación objetiva, racional y ponderada, no es suficiente una convocatoria general a cualquier persona, como la hecha en este asunto, sino que resulta necesario el llamado efectivo de quienes hacen parte del grupo o sector afectado con el Reglamento Técnico. 
Atendiendo a la consideraciones antes expuestas, concluye la Sala que se vulneraron los parámetros establecidos para la adopción del mencionado reglamento técnico, "los cuales no constituyen una mera formalidad en la expedición del acto administrativo sino un requisito sustancial para la efectividad del derecho a la participación de los afectados con decisiones como esta", y por ende anula la resolución al prosperar el cargo mencionado.

\subsection{Auto del 15 de febrero de 2016, proceso con radicación 11001-03-27-000-2016-00008-00, M. Sustanciador: Hugo Fernando Bastidas Bárcenas, Sección Cuarta del Consejo de Estado}

En este proceso se pretende la nulidad de la Resolución 5358 de 2015 expedida por el Ministerio de Transporte y "Por la cual se establece la base gravable de los vehículos automotores para la vigencia fiscal 2016". Entre otras disposiciones supuestamente vulneradas por la resolución, se citó por el demandante el artículo 8.8 CPACA y el Decreto 1345 de 2010, al considerarse que su expedición fue irregular, en tanto no se cumplió con el deber de "publicar" y "socializar" su proyecto. Con fundamento en estos hechos se fundamentó la solicitud de la medida cautelar de urgencia de suspensión provisional.

Tras repasar el marco normativo que rige la medida cautelar de urgencia en el CPACA, el magistrado sustanciador consideró sobre al cargo antes descrito que, según el artículo 8.8 de la Ley 1437 de 2011, contenidos regulatorios como los establecidos en la Resolución 5358 deben someterse a consideración del público y por eso tendría que haberse asegurado la respectiva divulgación y haberse fijado los plazos para oír a los interesados: contribuyentes, entidades territoriales, asociaciones gremiales, etc. En este sentido señala expresamente:

La Sala unitaria deduce de la sola lectura de los considerandos de la Resolución 5358 que no fue objeto de ninguna divulgación previa, hecho que habría constituido una violación por falta de aplicación de las dos normas que se acaban de reseñar. Otra circunstancia más que constituiría, en principio, un caso de nulidad del acto por expedición de forma irregular.

Con el razonamiento antes expuesto, entre otros argumentos, decretó la Sala Unitaria la medida cautelar urgente de suspender de forma provisional y preventiva la Resolución 5358 de 2015 proferida por el Ministerio de Transporte; al momento de la presentación de este artículo el proceso aún se encuentra pendiente de fallo definitivo. 


\subsection{Sentencia del 21 de julio de 2016, proceso con radicado} 11001-03-28-000-2015-00030-00, C.P.: Alberto Yepes Barreiro, Sección Quinta del Consejo de Estado

A través del medio de control de nulidad se demandó por un ciudadano el Decreto 2033 de 2015 por medio del cual el Gobierno Nacional dictó normas para la conservación del orden público durante el período de elecciones de autoridades y corporaciones públicas del 25 de octubre de 2015. El cargo principal endilgado se refiere a que en la expedición del mismo se pretermitió a los ciudadanos la oportunidad de ejercer su derecho a participar en la toma de la decisión, pese a existir, en su criterio, el deber de consulta y publicidad antes de expedir y publicar el decreto. Lo anterior se sustentó citando principalmente al artículo 8.8 cPACA y el Decreto 1345 de 2010.

Una vez transcritas las normas invocadas como vulneradas, consideró la Sala que de ellas "no se desprende de manera clara y expresa el deber de que los proyectos de decretos, en particular el Decreto 2033 de 2015 fuera sometido a consideración de la ciudadanía con el fin de que participara en su expedición". Para sustentar dicha afirmación analiza la Sala el marco constitucional vigente, las normas del bloque de constitucionalidad y la jurisprudencia de la Corte Constitucional, concluyendo que de este marco no se desprende la obligación de someter a consideración los proyectos de decreto. A continuación desciende al plano legal, y al analizar el contenido del artículo 8.8 CPACA concluye tras un ejercicio hermenéutico "sistemático" que el deber de publicar allí referido se impone "únicamente respecto de aquellos proyectos que orientan u organizan las relaciones entre la autoridad administrativa y los ciudadanos y no a aquellos actos de carácter general que expide en desarrollo de sus labores misionales".

Por último señala la Sala que "resultaría contrario a todo orden que la administración, para el desarrollo de sus funciones constitucionales y legales, en lo que toca al ejercicio de sus labores misionales, deba consultar cada una de sus actuaciones con la ciudadanía pues ello redundaría en un desquiciamiento de las potestades que el ordenamiento confiere para que estas sean desarrolladas conforme los principios que gobiernan la función pública". A partir de lo anterior, la Sala procede a negar las pretensiones de nulidad del decreto demandado.

A manera de balance preliminar sobre las providencias antes referenciadas, pueden destacarse al menos los dos siguientes puntos. En primer lugar, y a pesar de ser un asunto incipiente como se señaló en la jurisprudencia del alto tribunal, sí llama la atención el carácter reiterado de los argumentos esgrimidos por las partes demandantes y los efectos jurídicos pretendidos con ellos, por lo que podría preverse que sea un asunto recurrente y relevante en litigios posteriores. En segundo lugar, deben destacarse las posiciones encontradas de las decisiones adoptadas por las distintas secciones, lo cual 
muestra lo discutible y relevante del tema y ameritaría la adopción de una posición unificada por el alto tribunal sobre el particular ${ }^{62}$.

Sin embargo, valga la pena destacar en este punto que, analizando los actos administrativos sujetos a control, puede establecerse una distinción entre los pronunciamientos analizados, ya que los dos primeros corresponden a actos expedidos en ejercicio de la potestad reglamentaria del Gobierno Nacional, artículo 189.11 superior, mientras que la Sentencia del 21 de julio de 2016 se refiere a un acto expedido en virtud de la función de policía administrativa que ostenta el Presidente de la República con fundamento en el artículo 189.4 constitucional. Lo cual, en nuestro criterio, será determinante para comprender el alcance de la consulta pública, como pasa a explicarse.

\section{PROPUESTAS DE ACTUACIÓN E INTERPRETACIÓN DE LA CONSULTA PÚBLICA A PARTIR DEL MARCO NORMATIVO VIGENTE}

A partir del marco normativo descrito y de los pronunciamientos judiciales de que sobre el tema se dispone, nos corresponde ahora evaluar los escenarios futuros de la consulta pública previa a la expedición de reglamentos. Para ello se propondrán dos escenarios de lege ferenda, propugnando por la necesidad de regulación legal de la figura y/o por la necesidad de unificar la jurisprudencia sobre el particular por parte del Consejo de Estado. Por último se propondrá una posible interpretación sistemática de lege data, a la luz de lo hasta ahora expuesto.

Así, en primer lugar, debemos señalar lo conveniente y oportuno que resultaría que el Congreso colombiano regulara, a través de una ley ordinaria, el procedimiento de consulta pública. En este sentido, los asuntos clave que, consideramos, deben ser objeto de regulación son: (i) establecer expresamente la obligatoriedad de este trámite para todas las autoridades públicas, incluyendo las del nivel descentralizado territorialmente; (ii) determinar plazos claros para aceptar comentarios, observaciones y sugerencias, asegurando que la consulta pública se acompañe no solo del proyecto, sino también de documentos de respaldo que brinden información sobre la decisión que se debate y estimulen la participación, tales como estudios previos y evaluaciones de impacto ex ante; (iii) explicitar el deber de retroalimentación por parte de las autoridades en plazos legalmente establecidos y garantizar que todos los comentarios recibidos se hagan públicos, al igual que sus respuestas; (iv) ordenar que las autoridades dispongan de procedimientos internos

62 Durante el proceso de edición del presente artículo, la Sala de Consulta y Servicio Civil expidió el concepto 2291 del 14 de septiembre de 2016, el cual se refiere al asunto objeto de investigación y el contenido del mismo no contradice las conclusiones aquí planteadas. Véase Consejo de Estado, Sala de Consulta y Servicio Civil. Concepto del 14 de septiembre de 2016, rad. 11001-03-06-000-2016-00066-00 (2291), C.P.: Édgar González López. 
claros para adelantar dichos procesos de consulta, incentivando el uso de herramientas virtuales y de acceso remoto. Para estos efectos se puede considerar las recomendaciones hechas por la OCDE como buenas prácticas sobre el particular ${ }^{63}$, las recomendaciones del CONPES, y por qué no, adoptar como punto de partida para la discusión el contenido al respecto del que fuera el Decreto-Ley 266 de 2000.

En este sentido no consideramos conveniente ni adecuado jurídicamente que dicha regulación se haga a través del ejercicio de la potestad reglamentaria, como parece sugerirse por parte del Documento CONPES 3816. La razón para ello es que un decreto de este contenido, por encontrarse en el mismo nivel normativo de aquellos otros expedidos en ejercicio de la potestad reglamentaria, no pareciera poder condicionar las formalidades en la expedición de otras normas de la misma jerarquía. Un decreto de estas características pareciera asimilarse a una especie de "decretos orgánicos" ${ }^{64}$, los cuales a pesar de haber existido son ajenos al sistema jurídico colombiano.

En segundo lugar, y ante la ausencia de ley que regule de forma precisa e integral el procedimiento de consulta pública de los proyectos de reglamentos, es conveniente que la Sala Plena del Consejo de Estado se pronuncie a través de una sentencia de unificación, en razón de la relevancia jurídica de la cuestión, y siente su posición al respecto. Lo anterior obtiene plena justificación por cuanto han sido distintas secciones las que han abordado el asunto, y al hacerlo han adoptado posiciones encontradas, lo cual redunda en la necesidad de adoptar una posición unificadora por parte de la Sala Plena del tribunal de cierre de la jurisdicción contencioso administrativa. En cuando al sentido del fallo que debería adoptarse por parte del alto tribunal, consideramos que este debe en cualquier caso ceñirse al marco jurídico vigente, en particular al parámetro de constitucionalidad establecido, que, como se ha dicho, tiene como rector al principio y derecho constitucional de la participación.

En tercer lugar, en ausencia de ley y de sentencia de unificación que defina el asunto, consideramos que con el marco normativo vigente la propuesta de interpretación de lege lata que debe hacerse sobre el particular debe tener en cuenta los siguientes aspectos:

(i) Cuando exista norma expresa que regule el procedimiento de expedición de ciertos reglamentos, incluyendo procedimientos de consultas públicas previas a su expedición, estos deberán seguirse estrictamente, como es el caso de los referidos a asuntos contables y financieros, los expedidos por las comisiones de regulación y las medidas sanitarias y fitosanitarias en el ámbito agroalimentario, tal y como se expuso anteriormente. Pretermitir dicha etapa de consulta pública podrá comprometer la validez del mencionado reglamento, tal y como lo ha sostenido el Consejo de Estado.

63 OCDE (2014), 104.

64 Quinche Ramírez y URRego Ortiz, ob. cit., 322. 
(ii) Para los demás, es claro que el artículo 8.8 CPACA plantea un deber legal para las autoridades de publicar los proyectos específicos de regulación, la información en que se fundamente y los plazos para recibir opiniones, sugerencias o propuestas alternativas. Sobre el alcance del término "regulación", valga señalar que como este no constituye un término definido legalmente, siguiendo un criterio gramatical y sistemático nos conduce a definirlo como aquel acto administrativo de contenido general y abstracto expedido con fundamento en el ejercicio de la potestad reglamentaria y con vocación de permanencia. Con esta interpretación se excluyen expresamente las normas dictadas con fundamento en la función de policía administrativa, ya que estos expresamente se encuentran excluidos del ámbito de aplicación del CPACA.

(iii) Las autoridades que reciban opiniones, sugerencias y propuestas alternativas en el término establecido deberán darle el tratamiento de derecho de petición a cada una de ellas en los términos de la Ley 1755 de 2015, y por ende darles respuesta en los términos establecidos, siempre que no superen al plazo otorgado en el proyecto de decreto. De ello se dejará registro público por parte de la entidad que deberá estar disponible para toda persona e incluirse en la memoria justificativa del reglamento. Si estas se reciben en plazos por fuera de los establecidos, igualmente deberá dárseles trámite como derecho de petición y habrán de responderse, pero no será ello condición para la expedición del reglamento. Igual sucedería con los comentarios de los comentarios, donde será determinante si alcanzaron a hacerse dentro del plazo establecido.

(iv) El procedimiento de consulta también deberá aplicarse para las modificaciones de los reglamentos vigentes, ya que no existe fundamento legal en contrario y además existe el riego de que se utilice este como mecanismo para eludir su obligatoriedad ${ }^{65}$.

(v) La omisión del deber legal de consulta pública compromete el elemento de validez del cumplimiento de las formalidades sustanciales del acto administrativo, y por su expedición en forma irregular se incurriría en causal de nulidad en los términos del artículo 137 CPACA. Este razonamiento además es confirmado por lo dispuesto por el artículo 46 CPACA.

Esta interpretación propuesta, por un lado, es plenamente compatible con el marco constitucional vigente, en particular con el principio participativo que inspira al Estado colombiano, con la dimensión de control del derecho a la participación y las disposiciones del bloque de constitucional que abogan por la participación ciudadana directa en los asuntos público, todo lo cual se acompasa con la jurisprudencia constitucional, en especial, con los mandatos de universalidad y expansibilidad del principio participativo que ha definido 
la Corte Constitucional en su doctrina. Por otro lado, existen razones de conveniencia que se han ido decantando en el derecho comparado, tal y como fueron antes expuestas y que se centran en destacar la mayor legitimidad, transparencia y eficacia de la que gozan aquellas regulaciones que han sido consultadas públicamente antes de ser expedidas.

Además esta interpretación no parece muy lejana de la posición de ciertas autoridades administrativa directamente implicadas en el asunto, como lo es la Presidencia de la República, la cual en el proceso judicial que dio lugar a la expedición de la sentencia del Consejo de Estado el 21 de julio del año en curso, en la contestación de la demanda y los alegatos de conclusión, reconoció expresamente que "[1]a ley obliga a la publicación de los proyectos de regulación, en temas como el ejercicio de la potestad reglamentaria, el ejercicio de las competencias de intervención en la economía o los mercados" $"$.

\section{CONCLUSIONES}

A manera de conclusiones puede señalarse que la figura de la consulta pública previa a la expedición de reglamentos tiene un significativo sustento en el derecho comparado. Ello queda demostrado al revisar los procedimientos para la expedición de regulaciones existentes en países como Estados Unidos y España, y en organizaciones como la Unión Europea, donde dentro de estos procedimientos, aunque con distintos alcances, queda establecida la necesidad de adelantar instancias de consulta pública previa a la expedición de estos tipos normativos. Además, organismos de cooperación multilateral como el Banco Mundial y la OCDE han venido sosteniendo y promocionando estas consultas públicas como buenas prácticas en el plano normativo nacional e internacional.

En segundo lugar, en relación con el régimen jurídico vigente de la consulta pública en Colombia, valga destacar que si bien en el plano constitucional no existe una norma que expresamente así lo exija, como sucede en el caso español, sí existen valores y principios que orientan la participación ciudadana, en decisiones administrativas que la afecten, al control de las actuaciones de la administración, y que orientan hacia la moralidad, imparcialidad y publicidad en el ejercicio de la función administrativa. Ya en el plano legal y reglamentario encontramos que para ciertos reglamentos existen requisitos de consulta pública en normas especiales que debería seguirse estrictamente, como es el caso de los referidos a asuntos contables y financieros, los expedidos por las comisiones de regulación y las medidas sanitarias y fitosanitarias en el ámbito agroalimentario. Ya de forma gené-

66 Consejo de Estado, Sección Quinta. Sentencia del 21 de julio de 2016, rad. 11001-0328-000-2015-00030-00, C.P.: Alberto Yepes Barreiro. 
rica para las demás "regulaciones técnicas" se encuentra lo dispuesto por el artículo 8.8 CPACA que dispone que los proyectos de las mismas deben mantenerse a disposición de toda persona, junto con la información en que se fundamenten y el plazo dentro del cual se podrán presentar observaciones, sugerencias o propuestas alternativas, de las cuales se dejará registro. Esta norma, en una interpretación sistemática del ordenamiento vigente, supone la existencia de un deber legal predicable de toda autoridad administrativa que tenga dentro de sus competencias la expedición de reglamentos, consistente en consultar públicamente los proyectos de reglamentos que pretenda adoptar, para lo cual deberá, dentro del plazo establecido, recibir, analizar y contestar, a la luz de la regulación estatutaria del derecho de petición, cada una de las intervenciones ciudadanas como requisito de validez de la expedición del reglamento.

Sin lugar a dudas, la generalidad e imprecisión que puede predicarse del artículo 8.8 CPACA hace conveniente la expedición de una ley que, como lo ha sugerido la OCDE, desarrolle precisamente la figura en un futuro cercano. De igual forma, y a raíz de pronunciamientos incipientes y aparentemente contradictorios por parte del Consejo de Estado al respecto, sería igualmente oportuno que el alto tribunal fijara una interpretación unificada sobre el alcance actual de la figura a la luz de un criterio sistemático y teleológico. Sin embargo, hasta que ello suceda, deberá ser el operador jurídico administrativo quien deberá ponderar entre los principios de eficacia, por un lado, y debido proceso administrativo-participación, por otro, para determinar el alcance y condiciones de la consulta pública antes de la expedición de un reglamento. El presente artículo pretendió brindarle algunos elementos para tener en consideración.

\section{REFERENCIAS}

Aberastury, P. y Blanke, H.-J., coords. (2011). Tendencias actuales del procedimiento administrativo en Latinoamérica y Europa. Buenos Aires: Eudeba.

Arboleda Perdomo, E. J. (2013). Comentarios al Nuevo Código de Procedimiento Administrativo y de lo Contencioso Administrativo. Bogotá: Legis.

BARnes, J., ed. (2008). La transformación del procedimiento administrativo. Sevilla: Derecho Global.

Benavides, J. L. (2013). ed. Código de Procedimiento Administrativo y de lo Contencioso Administrativo. Bogotá: Universidad Externado de Colombia.

Consejo de Estado, Sección Primera. Sentencia del 4 de febrero de 2016, rad. 11001-0324000-2010-00329-00, C.P.: Guillermo Vargas Ayala.

Consejo de Estado, Sección Cuarta. Auto del 15 de febrero de 2016, rad. 11001-03-27-0002016-00008-00, M. sustanciador: Hugo Fernando Bastidas Bárcenas. 
Consejo de Estado, Sección Quinta. Sentencia del 21 de julio de 2016, rad. 11001-03-28000-2015-00030-00, C.P.: Alberto Yepes Barreiro.

Consejo de Estado, Sala de Consulta y Servicio Civil. Concepto del 14 de septiembre de 2016, rad. 11001-03-06-000-2016-00066-00 (2291), C.P.: Édgar González López.

Cornelius, K. (1999). Rulemaking: How Government Agencies Write Law and make Policy,

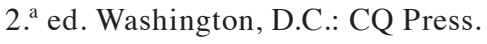

Corte Constitucional. Sentencia C-089 de 1994, M.P: Eduardo Cifuentes Muñoz.

Corte Constitucional. Sentencia C-150 de 2015, M.P: Mauricio Gonzalez Cuervo.

Corte Constitucional. Sentencia C-225 de 1995, M.P: Alejandro Martínez Caballero.

Documento CONPES 3816: Mejora normativa: análisis de impacto, disponible en: https:// colaboracion.dnp.gov.co/CDT/Conpes/Econ\%C3\%B3micos/3816.pdf

García de Enterría, E. y Fernández, T.-R. (2000). Curso de derecho administrativo, 10. ${ }^{2}$ ed., Madrid, Civitas.

Islam, R. (2003). Do more transparent Governemnt Govern Better? Washington D.C., Banco Mundial.

López Medina, Diego. (2008). "Prólogo”. En Peña Porras, D. I. La construcción del derecho administrativo colombiano. Bogotá, Universidad de los Andes y Temis.

McNollgast, H. (1999). "The political origins of the Administrative Procedure Act". The Journal of Law, Economics \& Organization, Oxford Journals. Vol. 15.

Montaña Plata, A. y Ospina Garzón, A. F., eds. (2014). La constitucionalización del derecho administrativo. Bogotá, Universidad Externado de Colombia.

OCDE, (2013 ). Colombia: Implementing good governance. Paris, OECD.

OCDE, (2014). Estudio de la OCDE sobre la política regulatoria en Colombia: más allá de la simplificación administrativa. Paris, OECD.

oECD, (2012). Recomendación del Consejo sobre Política y Gobernanza Regulatoria. Paris, OCDE. .

Quinche Ramírez, M. F. y Urrego Ortiz, F. (2011). Los decretos presidenciales en el sistema normativo. Bogotá, Doctrina y Ley.

Real Academia de la Lengua, Diccionario de la Lengua Española.

Rosenbloom, D. H. (2003). Administrative law for public managers. Washington D.C., Westview Press, .

SÁnchez Morón, M. (2012 ). Derecho administrativo: Parte general, Madrid, Tecnos.

SÁnchez, V. M. (2012). Derecho de la Unión Europea, Madrid, Huygens.

Unión Europea. (2010). Normativa inteligente: documento de síntesis. Bruselas, Unión Europea. 
Xanthaki, H. (2014). EU Legislative Quality post-Lisbon: the challeges of Smart Regulation. En Statute Law Review, 35.

Xanthaki, Helen. Drafting Legislation: Art and Technology of Rules for Regulation. London, Hart Publishing, 2014. 